\title{
Imaging Multiple Colloidal Particles by Fitting Electromagnetic Scattering Solutions to Digital Holograms
}

\section{Citation}

Fung, Jerome, Rebecca W. Perry, Thomas G. Dimiduk, and Vinothan N. Manoharan. 2012. "Imaging Multiple Colloidal Particles by Fitting Electromagnetic Scattering Solutions to Digital Holograms." Journal of Quantitative Spectroscopy and Radiative Transfer 113 (18) (December): 2482-2489. doi:10.1016/j.jqsrt.2012.06.007.

\section{Published Version}

doi:10.1016/j.jqsrt.2012.06.007

\section{Permanent link}

http://nrs.harvard.edu/urn-3:HUL.InstRepos:24889103

\section{Terms of Use}

This article was downloaded from Harvard University's DASH repository, and is made available under the terms and conditions applicable to Other Posted Material, as set forth at http:// nrs.harvard.edu/urn-3:HUL.InstRepos:dash.current.terms-of-use\#LAA

\section{Share Your Story}

The Harvard community has made this article openly available.

Please share how this access benefits you. Submit a story.

Accessibility 


\title{
Imaging Multiple Colloidal Particles by Fitting Electromagnetic Scattering Solutions to Digital Holograms
}

\author{
Jerome Fung ${ }^{\mathrm{a}}$, Rebecca W. Perry ${ }^{\mathrm{b}}$, Thomas G. Dimiduk ${ }^{\mathrm{a}}$, Vinothan \\ N. Manoharan ${ }^{\mathrm{b}, \mathrm{a}, *}$ \\ ${ }^{a}$ Dept. of Physics, Harvard University, 17 Oxford Street, Cambridge, MA 02138, USA \\ ${ }^{b}$ School of Engineering 83 Applied Sciences, Harvard University, 29 Oxford Street, \\ Cambridge, MA 02138, USA
}

\begin{abstract}
Digital holographic microscopy is a fast three-dimensional (3D) imaging tool with many applications in soft matter physics. Recent studies have shown that electromagnetic scattering solutions can be fit to digital holograms to obtain the 3D positions of isolated colloidal spheres with nanometer precision and millisecond temporal resolution. Here we describe the results of new techniques that extend the range of systems that can be studied with fitting. We show that an exact multisphere superposition scattering solution can fit holograms of colloidal clusters containing up to six spheres. We also introduce an approximate and computationally simpler solution, Mie superposition, that is valid for multiple spheres spaced several wavelengths or more from one another. We show that this method can be used to analyze holograms of several spheres on an emulsion droplet, and we give a quantitative criterion for assessing its validity.
\end{abstract}

Keywords: light scattering, digital holography, colloids, emulsions

\footnotetext{
*Corresponding author

Email addresses: fung@physics.harvard.edu (Jerome Fung), vnm@seas.harvard.edu (Vinothan N. Manoharan)
}

NOTICE: this is the author's version of a work that was accepted for publication in the Journal of Quantitative Spectroscopy and Radiative Transfer. Changes resulting from the publishing process, such as peer review, editing, corrections, structural formatting, and other quality control mechanisms may not be reflected in this document. Changes may have been made to this work since it was submitted for publication. A definitive version was subsequently published in Journal of Quantitative Spectroscopy and Radiative Transfer 113(18): 2482 (2012). 


\section{Introduction}

In-line digital holographic microscopy (DHM) has emerged as a powerful and useful 3-dimensional (3D) imaging technique for characterizing colloids and soft materials $[1,2,3,4,5,6,7]$. Unlike conventional microscopes, holographic microscopes use a coherent source to illuminate a sample, generating a two-dimensional interference pattern, or hologram, that encodes 3D information (Figure 1a). For relatively simple objects, such as colloidal spheres, the most precise way to recover this $3 \mathrm{D}$ information is to fit an electromagnetic scattering solution such as the Lorenz-Mie solution to the hologram. The fitting technique can reveal the positions of particles with nanometerscale spatial precision [1] on sub-millisecond time scales. The high temporal resolution, more than an order of magnitude larger than that of other 3D imaging techniques such as confocal microscopy, has enabled observations of new physical phenomena, as demonstrated in a recent study [7] showing the detailed dynamics of a colloidal particle interacting with an oil-water interface.

Thus far, the fitting technique has been used only to analyze holograms of simple scatterers. The seminal work of Lee et al. [1] as well as recent experiments from Kaz et al. [7] examined single isolated spheres, for which the well-known Lorenz-Mie solution suffices. Recently Fung et al. used a multisphere superposition code [8] to model digital holograms of non-rigid colloidal sphere dimers and rigid trimers [6]. To date, however, there have been no reports on using scattering solutions to analyze holograms of more complex scatterers, including clusters of more than 3 particles or colloidal systems with configurations that are unknown a priori.

Such systems are of significant physical interest. Recently, Meng et al. showed how clusters of six or more spheres with a short-ranged attraction selfassemble into morphologies governed by rotational and vibrational entropy [9]. Detailed 3D observations of the self-assembly process and transitions between different cluster morphologies were not possible in that work, which relied on conventional microscopy. Another interesting system consists of colloidal particles bound to the surface of an emulsion droplet. The particle configurations, which need not be rigid or symmetrical [10], may reveal information about the still poorly-understood interaction forces between colloidal particles on a curved liquid-liquid interface [11].

In this work we demonstrate new fitting methods for analyzing digital holograms of more complex scatterers, including rigid clusters containing up 
to 6 particles and nonrigid configurations of particles bound to the surface of an emulsion droplet. We fit two analytical scattering solutions to our experimental data: an exact multisphere superposition solution and an approximate, computationally simpler approximation, Mie superposition. Both solutions can be used to compute holograms from an arbitrary number of spherical particles in an arbitrary geometry, but they differ in the precision to which they can fit holograms of the two experimental systems. We discuss the applicability of the Mie superposition approximation and the challenges remaining in the application of these techniques to other colloidal systems.

\section{Experimental Methods}

\subsection{Sample Preparation}

We image two different types of samples in this work: colloidal clusters bound by depletion forces, and particles bound to the surface of indexmatched emulsion droplets.

\subsubsection{Colloidal Clusters}

We manually assemble tetrahedra containing four spheres, trigonal bipyramids containing five spheres, and polytetrahedra containing six spheres using the optical trap described in Section 2.2. Bright-field micrographs of these clusters are shown in Figure 2. To make the clusters, we first prepare a dilute suspension of monodisperse, $1.3-\mu$ m-diameter surfactant-free, sulfatestabilized polystyrene spheres (Invitrogen) at a volume fraction of $8 \times 10^{-6}$ in an aqueous solution containing $5 \mathrm{mM} \mathrm{NaCl}$ and $246 \mathrm{mM}$ sodium dodecyl sulfate (SDS). We load this suspension into sample cells made from untreated glass, as described in [6]. Because the SDS concentration is far above the critical micelle concentration [12], the SDS forms micelles which induce a short-ranged depletion attraction between the polystyrene spheres [13, 14]. This attraction causes spheres to bind together into clusters when drawn into the focus of the optical trap. In all cases, we verify the cluster geometry by direct observation with bright-field microscopy before holographic imaging.

\subsubsection{Particles on Emulsion Droplets}

We prepare emulsion droplets laden with colloidal spheres by dispersing a suspension of microspheres in oil into an aqueous continuous phase. We suspend $0.8 \mu \mathrm{m}$ diameter polymethyl methacrylate (PMMA) spheres coated with poly(12-hydroxystearic acid) stabilizer $[15,16]$ in decane at a 

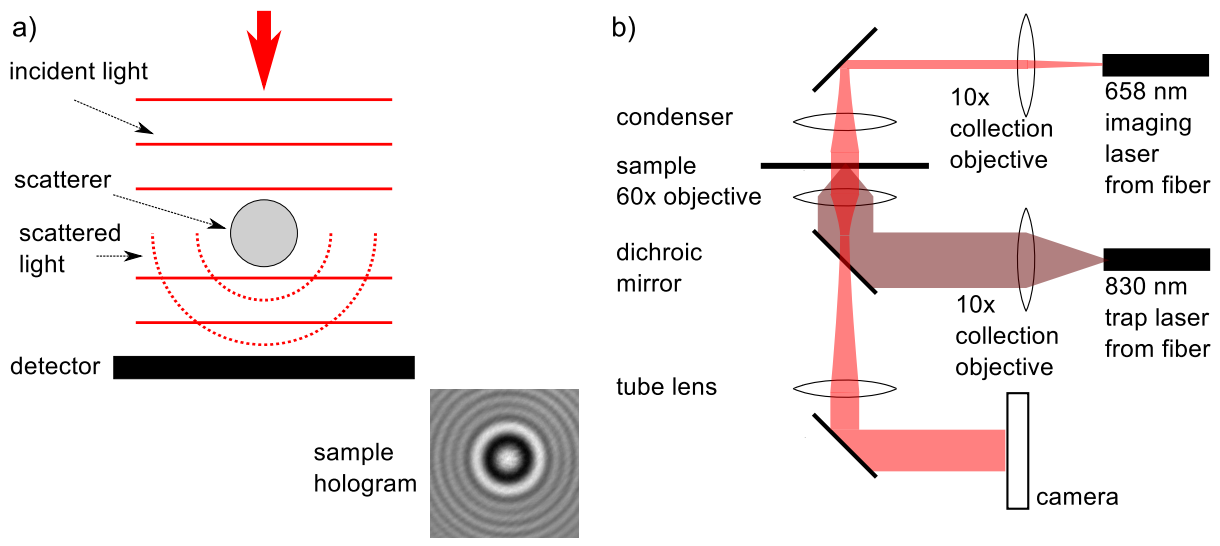

Figure 1: a) Schematic illustration of in-line digital holographic microscopy. An incident plane wave illuminates a scatterer, and a detector records the interference pattern formed by the scattered light and unscattered incident light. The inset shows a typical interference pattern, or hologram, formed by a single 1- $\mu$ m-diameter polystyrene sphere in water. b) Schematic illustration of DHM optical train, showing the counterpropagating laser beams used for DHM (lighter red) and optical trapping (darker red).
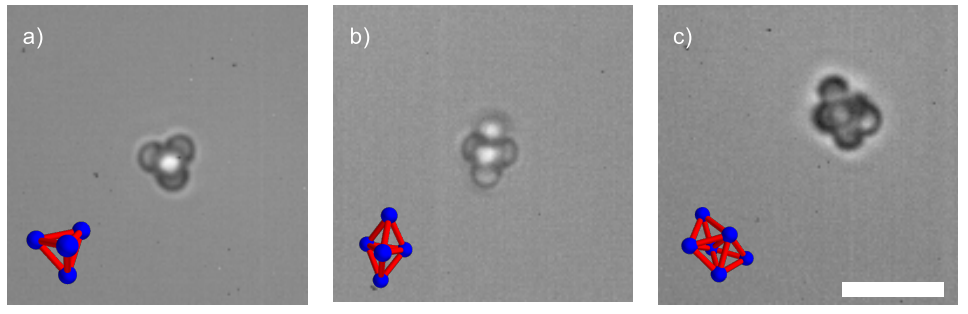

Figure 2: Bright field micrographs of clusters made from 1.3- $\mu$ m diameter polystyrene spheres, recorded with a $60 \times 1.20 \mathrm{NA}$ water immersion objective. Ball-and-stick models, indicating cluster geometry and orientation, are a guide to the eye. Scale bar $5 \mu \mathrm{m} . n_{s}$ denotes the number of spheres in each cluster. a) Tetrahedron $\left(n_{s}=4\right)$. b) Trigonal bipyramid $\left(n_{s}=5\right)$. c) Polytetrahedron $\left(n_{s}=6\right)$. 
mass fraction of $2.1 \times 10^{-3}$. The aqueous continuous phase contains $0.2 \%$ $\mathrm{w} / \mathrm{w}$ Pluronic P123 triblock copolymer surfactant and $56 \% \mathrm{w} / \mathrm{w}$ glycerol. The glycerol matches the refractive index of the continuous phase to that of decane, so that only the PMMA spheres scatter light. We prepare the emulsions by mixing $0.5 \mathrm{~mL}$ of the PMMA-containing decane with $20 \mathrm{~mL}$ of the continuous phase in a $40 \mathrm{~mL}$ scintillation vial and shearing the mixture for 3 minutes at $9500 \mathrm{rpm}$ with an Ika T9 Basic homogenizer equipped with a S25N-8G dispersing tool. After emulsification, we dilute the emulsion to $17 \% \mathrm{v} / \mathrm{v}$ with additional continuous phase. A micrograph of a typical emulsion droplet, laden with 4 particles but with the continuous phase slightly mismatched to allow the droplet to be seen, is shown in Figure 3.

In order to minimize both the effects of nearby glass surfaces on the interparticle interactions and unwanted back-reflections of the scattered light during imaging, we use negative dielectrophoresis (NDEP) to keep the particleladen decane droplets away from the top of our sample cells, to which they would otherwise rise due to buoyancy. We prepare sample cells similar in design to those used for the cluster measurements, except that the surface of the uppermost glass microscope slide in contact with the emulsion contains a linear array of interdigitated indium tin oxide (ITO) electrodes with a 40 $\mu \mathrm{m}$ spacing between adjacent electrodes $[17,18,19]$. By applying a $10 \mathrm{~V}$ peak-to-peak, $300 \mathrm{kHz}$ square wave with an arbitrary waveform generator (Agilent AFG 3022B), we produce a downward NDEP force on the droplets. Diffraction from the edges of the ITO electrodes is negligible.

We use photolithography and wet etching to prepare the interdigitated electrode arrays on microscope slides coated with a $30 \mathrm{~nm}$ layer of ITO (Delta Technologies, CB-90IN coating). We spin-coat the ITO surface of the slides with Shipley S1813 positive photoresist at $5000 \mathrm{rpm}$ and soft bake the slides on a $115^{\circ} \mathrm{C}$ hot plate for 1 minute. We first define the electrode pattern by exposing the photoresist to UV light through a photomask $\left(150 \mathrm{~mJ} / \mathrm{cm}^{2}\right.$ exposure at $405 \mathrm{~nm}$ ), then develop the photoresist by immersion in Microposit MF CD-26 developer for 1 minute at room temperature. Following an overnight hard bake in a $90^{\circ} \mathrm{C}$ oven, we etch away the exposed ITO with an aqueous solution containing $40 \% \mathrm{v} / \mathrm{v} \mathrm{HCl}$ and $10 \% \mathrm{v} / \mathrm{v} \mathrm{HNO}_{3}$ for 12 minutes at room temperature.

\subsection{Recording Digital Holograms}

We record digital holograms on a modified Nikon inverted microscope (Figure 1b). Our apparatus is described in detail in $[6,7]$. In brief, we 
couple a $660 \mathrm{~nm}$ laser diode (Opnext HL6545MG) into a single-mode fiber, from which we obtain up to $60 \mathrm{~mW}$ of laser power. The beam is collimated by the microscope's condenser before striking the sample. To image colloidal clusters, we use a $60 \times, 1.20 \mathrm{NA}$ water immersion objective (Nikon). To image particles on droplets, we use a $100 \times, 1.40$ NA oil immersion objective (Nikon) with an immersion oil of index 1.412 (Cargille). In both cases, the index of refraction of the immersion medium is chosen to match that of the continuous phase of the sample, thus minimizing height-dependent spherical aberration [20]. Images are recorded by a Photon Focus MVD-1024E-160 camera and captured to disk by an EPIX PIXCI E4 frame grabber.

We also integrate an optical trap into the apparatus to manually assemble colloidal clusters and manipulate them. The trap beam is kept off during holographic imaging. Light from the $830 \mathrm{~nm}$ trap diode (Sanyo DL-8142201 ) is filtered through a single-mode fiber and reflected by a dichroic mirror into the back aperture of the Nikon objective lens used for imaging, as shown in Figure 1b.

\section{Fitting Scattering Solutions to Digital Holograms}

\subsection{Pre-Processing Holograms \& Fitting Scattering Models}

After recording digital holograms and suitable backgrounds in an experiment, we fit scattering models to the holograms. We have released our software tools for doing so in the open-source package Holopy, available at https://launchpad.net/holopy. As described in detail in [6], we divide the recorded holograms by a background taken in an empty field of view and normalize them to a mean value of 1 . We then use the Levenberg-Marquardt algorithm to minimize the sum of residuals between the experimental holograms and model holograms computed from a set of fixed and variable physical parameters, including particle coordinates, sizes, and refractive indices. We discuss the models in more detail in Section 3.2.

Two features of the Levenberg-Marquardt iterative minimization algorithm have significant implications for our analysis. First, the algorithm requires initial values for all the model parameters. Typically, we choose these initial values to provide a qualitative match to the experimentally recorded holograms, allowing the algorithm to converge after approximately 10 iterations. Second, the algorithm requires the number of spheres in the hologram to be known a priori. When necessary, we can determine the num- 
ber of spheres using complementary hologram analysis techniques, such as Rayleigh-Sommerfeld reconstruction [21].

We choose the parameters in the scattering models to obtain as much physical information from the holograms as possible while minimizing the required computational time. When modeling holograms of rigid clusters, we assume that all of the spheres in the clusters have the same radius and real refractive index, and that the gap between spheres is uniform. We vary the common radius, the common particle refractive index, the common gap distance, the cluster center of mass, the three orientational Euler angles, and the scaling coefficient $\alpha$ (see equation 1 ). When modeling holograms of particles on an emulsion droplet, we vary the common particle radius, the common particle refractive index, 3 spatial coordinates per particle, and the scaling coefficient $\alpha$. While we could use models with more parameters, such as arbitrary radii for all the spheres, we gain little useful information from doing so, since our spheres are monodisperse. Fitting more complex models requires significantly more computation time, since the time required to fit a model with $N_{\text {params }}$ parameters scales as $N_{\text {params }}^{2}$.

\subsection{Modeling Holograms}

In our models, the normalized hologram $I_{h o l}$ may be described as

$$
I_{h o l}=1+2 \alpha \Re\left[\mathbf{E}_{\text {scat }} \cdot \hat{\mathbf{e}}\right]+\alpha^{2}\left|\mathbf{E}_{\text {scat }}\right|^{2}
$$

where $\mathbf{E}_{\text {scat }}$ is the scattered electric field, and $\hat{\mathbf{e}}$ is the unit vector describing the incident polarization. As described in $[1,6], \alpha$ is inversely proportional to the magnitude of the incident electric field $\mathbf{E}_{\text {inc }}$ and allows us to model variations in the incident laser intensity. In our experiments, we know the optical parameters of the system, including the medium refractive index, the incident laser wavelength, and the apparent detector pixel size (after magnification). Therefore, we require only an algorithm for computing $\mathbf{E}_{\text {scat }}$ to model a hologram of some given configuration of particles. Any such algorithm must include the full radial dependence of $\mathbf{E}_{\text {scat }}$ on spherical Hankel functions of the first kind and their derivatives, as the far-field asymptotic approximation does not hold for the distances at which we record holograms [6].

We use two analytical methods to calculate $\mathbf{E}_{\text {scat }}$ for multiple spheres. The first method, described in detail in [6], uses the multisphere superposition code SCSMFO developed by Mackowski et al. [8]. Previously we termed this 
approach the "T-matrix method", but we now prefer the term "multisphere superposition" since we never actually compute the $T$-matrix itself, which is typically used for orientational averaging. This method gives exact numerical solutions to Maxwell's equations for the scattering of a plane wave by multiple spheres, accounting for all electromagnetic coupling between the particles $[8,22]$.

The second approach is to superpose the fields calculated from the LorenzMie solution for each of the spheres, taking into account the phase differences arising from the displacement of the spheres along the optical axis. This approach, which we term Mie superposition, assumes that only a plane wave falls onto each sphere. It neglects electromagnetic coupling, including multiple scattering, between the spheres. $\mathbf{E}_{\text {scat }}$ calculated from Mie superposition is the lowest-order approximation to multisphere superposition; it is the initial solution that SCSMFO iteratively corrects in the order-of-scattering approach [23].

The main advantage of Mie superposition over multisphere superposition is that it is less computationally costly for multiple spheres that are spaced far apart. SCSMFO expresses $\mathbf{E}_{\text {scat }}$ as a superposition of vector spherical harmonics whose origin lay at the center of mass of all the particles. It does so using vector spherical harmonic translation theorems to translate field expansions centered about each sphere to the cluster center of mass [8]. When the particles are far from their center of mass, the final field expansion contains many more terms than would be necessary in a field expansion centered about the individual spheres. We therefore use the Mie superposition approach for our submicron-diameter PMMA particles on emulsion droplets, which are weakly scattering (size parameter $x \approx 5$ and relative index $m \approx 1.07$ ) and spaced far apart. We discuss the validity of Mie superposition further in Section 4.3.

After fitting a scattering model to a hologram, we quantitatively confirm the fit of the model by examining two statistical measures of the goodnessof-fit. The first measure, chi-squared per pixel $\chi_{p}^{2}$, is the quantity the fitting algorithm attempts to minimize:

$$
\chi_{p}^{2}=\frac{1}{N} \sum_{i=1}^{N}\left(I_{\text {holo }}-I_{f i t}\right)^{2} .
$$

The sums run over all $N$ pixels of the recorded normalized hologram $I_{\text {holo }}$ and the best-fit model hologram $I_{f i t}$. For any given hologram, comparing $\chi_{p}^{2}$ 
to an expected noise level allows us to assess whether deviations between the recorded hologram and best-fit model are due to instrumental noise or to a systematic error in the model's description of the underlying data. Assuming noise in the least significant bit of an 8-bit camera, we would expect $\chi_{p}^{2}$ values greater than $(1 / 255)^{2}=1.54 \times 10^{-5}$ to originate from systematic errors.

The second statistical measure we use is the coefficient of determination $R^{2}$. We define $R^{2}$ as

$$
R^{2}=1-\frac{\sum_{i=1}^{N}\left(I_{\text {holo }}-I_{\text {fit }}\right)^{2}}{\sum_{i=1}^{N}\left(I_{\text {holo }}-\bar{I}_{\text {holo }}\right)^{2}}=1-\frac{\sum_{i=1}^{N}\left(I_{\text {holo }}-I_{\text {fit }}\right)^{2}}{\sum_{i=1}^{N}\left(I_{\text {holo }}-1\right)^{2}}
$$

where $\bar{I}_{\text {holo }}$ is the mean value of the recorded hologram, which is 1 by our normalization [24]. $R^{2}$ measures the fraction of the variation of the recorded hologram from its mean value that is captured by the best-fit model, independent of the amount of variation in the hologram. Whereas $\chi_{p}^{2}$ varies significantly across physical systems that differ in scattering cross section and hence hologram fringe amplitude, $R^{2}$ does not. Therefore, we use $R^{2}$ to assess the validity of the scattering models fitted to the holograms. In particular, as we discuss in Section $4.3, R^{2}$ helps to assess the validity of the Mie superposition approximation.

\section{Results and Discussion}

\subsection{Rigid Clusters}

Figure 4 compares recorded and best-fit model holograms, calculated with multisphere superposition, for tetrahedral, trigonal bipyramidal, and polytetrahedral clusters. Qualitatively, we observe excellent agreement between the recorded and best-fit holograms. In particular, the best-fit models reproduce the highly non-axisymmetric fringes in the recorded holograms, which depend strongly on the cluster orientations. The quality of the agreement is confirmed by the $R^{2}$ values of the fits, which are close to 1 (Table 1 ). Also, the fitted particle radii are close to the manufacturer's reported value of 650 $n m$. However, the values of $\chi_{p}^{2}$ we observe are an order of magnitude larger than what we would expect due to camera noise, and indicate that further improvements to fits will depend on modeling additional physical phenomena or improving the convergence of the fitter. 


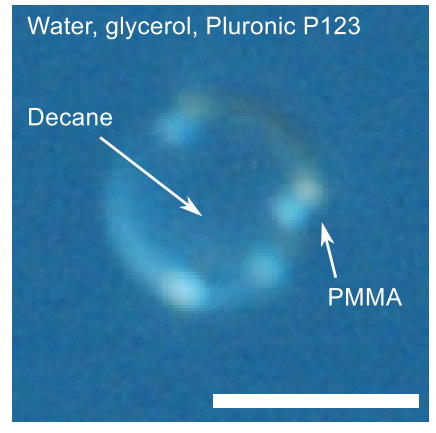

Figure 3: Bright field micrograph of decane droplet with 4 PMMA spheres on its surface, recorded under 100× magnification and differential interference contrast (DIC). Here, a slight index mismatch between the decane and the continuous phase makes the droplet visible under DIC. Scale bar $5 \mu \mathrm{m}$.

\begin{tabular}{lccc} 
Cluster & Radius $(\mathrm{nm})$ & $\chi_{p}^{2}$ & $R^{2}$ \\
\hline Tetrahedron & $670 \pm 30$ & $1.18 \times 10^{-3}$ & 0.923 \\
Trigonal bipyramid & $640 \pm 30$ & $9.48 \times 10^{-4}$ & 0.910 \\
Polytetrahedron & $650 \pm 20$ & $1.24 \times 10^{-3}$ & 0.877
\end{tabular}

Table 1: Fitted radii and goodness-of-fit statistics $\chi_{p}^{2}$ and $R^{2}$ for rigid cluster holograms in Figure 4. 

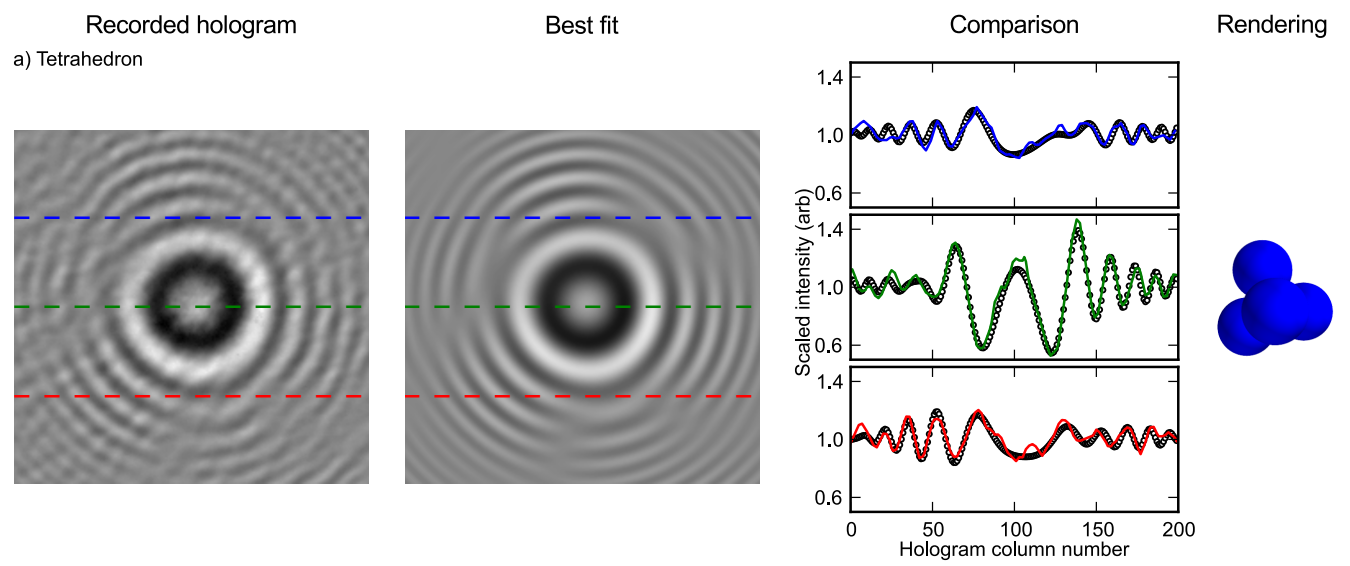

b) Trigonal bipyramid
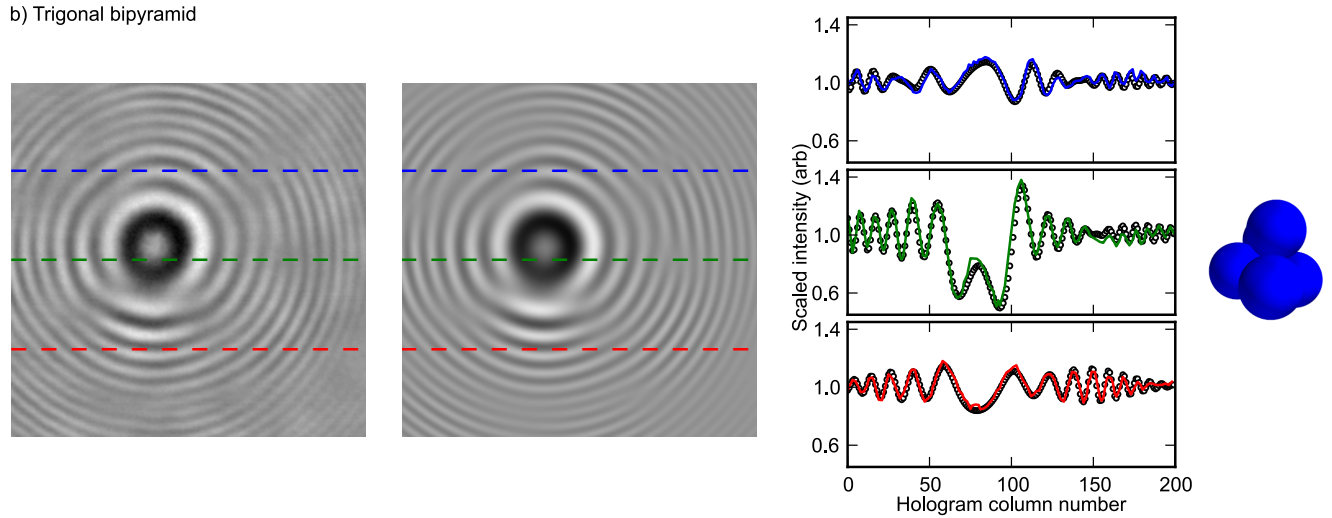

c) Polytetrahedron
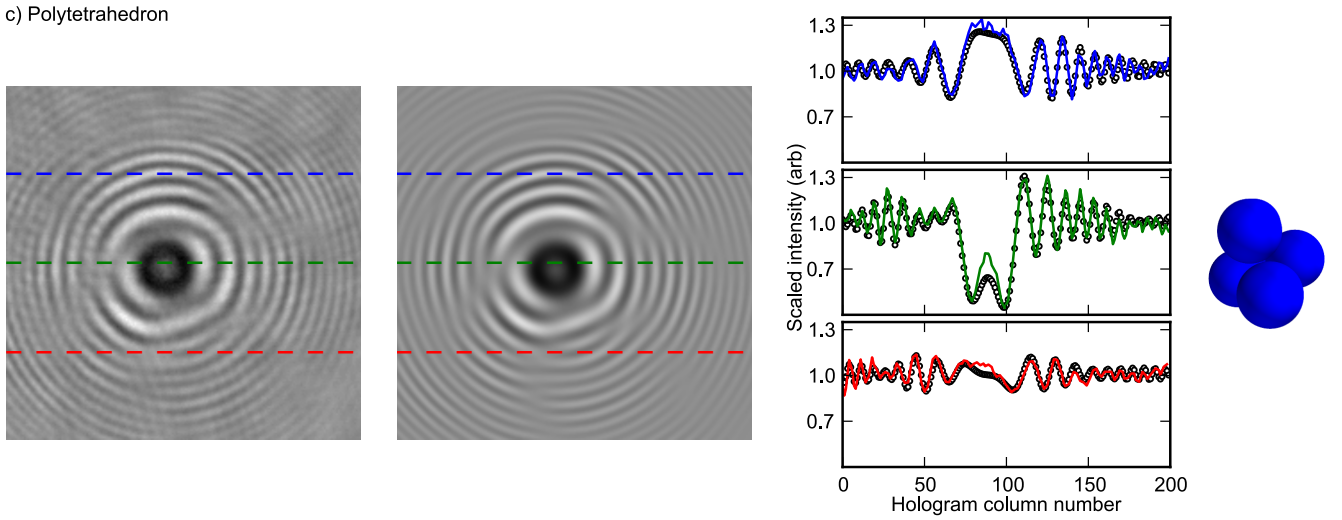

Figure 4: Recorded and best-fit model holograms of rigid clusters. The third column compares the recorded holograms (solid lines) to the best-fit models (open symbols) along the color-coded dashed horizontal lines shown in the holograms. The renderings in the rightmost column show the cluster orientations determined from the fits. In the renderings, the incident light propagates into the page. a) Tetrahedron $\left(n_{s}=4\right)$. b) Trigonal bipyramid $\left(n_{s}=5\right)$. c) Polytetrahedron $\left(n_{s}=6\right)$. 

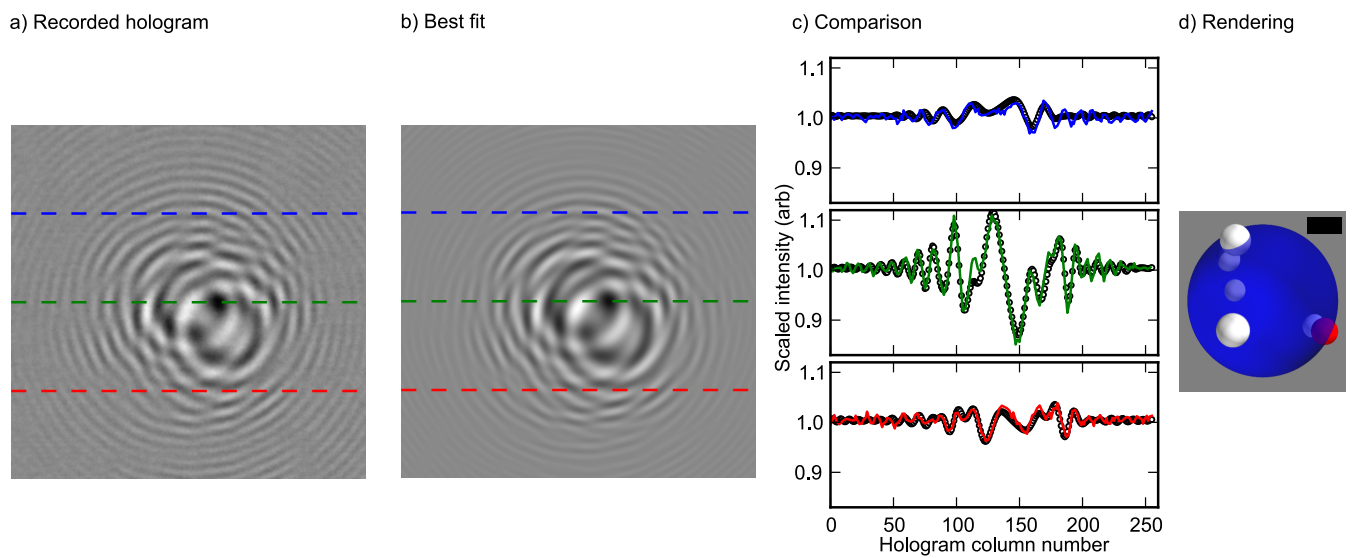

Figure 5: Holograms of six PMMA spheres on a decane droplet. a) Recorded hologram. b) Best-fit model. c) Comparison between recorded hologram (solid lines) and best-fit model computed using Mie superposition (open symbols) along the dashed horizontal lines in the holograms. d) Rendering showing the sphere positions determined by fitting the holograms. The incident light propagates into the page, and the scale bar is $1 \mu \mathrm{m}$. The larger blue sphere is a guide to the eye; its position and diameter, $4.35 \mu \mathrm{m}$, were determined by fitting a sphere to the coordinates of the six particles. The small red sphere indicates the particle showing the largest discrepancy in position along the optical axis between fits to Mie superposition and multisphere superposition. 


\subsection{Particles on Droplets}

Here we show that our fitting techniques may also be applied to multisphere systems without a fixed geometry. Figure 5 shows a hologram of six particles bound to the surface of a decane droplet and a best-fit model calculated using Mie superposition. Again, the qualitative agreement between the fringes of the recorded hologram and the best-fit model is good. Quantitatively, we find $\chi_{p}^{2}=7.29 \times 10^{-5}$ and $R^{2}=0.811 . \chi_{p}^{2}$ is much lower than the values we obtained for the clusters, primarily because the peak amplitude of the hologram in Figure 5 is significantly smaller than the peak amplitudes of the cluster holograms in Figure 4 . The value of $R^{2}$ indicates that the fit is slightly worse than the fits for the clusters. However, because we know the particles are bound to the surface of a spherical droplet, we can independently test the accuracy of the fitted particle positions. While we cannot directly image the decane droplet, which is index-matched to the continuous phase, we can fit the surface of a sphere to the particle coordinates, as shown in Figure 5d. The average difference between the radial distance of each particle from the droplet center and the fitted droplet radius is $60 \pm 60$ $\mathrm{nm}$. Differences of this scale are comparable to previously reported precisions for DHM $[1,6]$, and may be partially accounted for by variations in the interfacial contact angle between different particles [7].

To determine whether the slightly worse value of $R^{2}$ obtained in this fit is due to the Mie superposition approximation, we examine the validity of this approximation in further detail.

\subsection{Applicability of Mie Superposition}

To confirm that Mie superposition is a suitable means for analyzing holograms like that in Figure 5, in which multiple weakly scattering spheres are situated several diameters apart, we fit a model based on multisphere superposition to the same hologram. The multisphere superposition fit yields $\chi_{p}^{2}=8.08 \times 10^{-5}$ and $R^{2}=0.790$, comparable to the Mie superposition fits. Differences between fitted particle coordinates in the two in-plane directions, perpendicular to the optical axis, are at most $22 \mathrm{~nm}$. The largest difference between fitted coordinates along the optical axis is $154 \mathrm{~nm}$. The sphere showing the largest displacement along the optical axis is highlighted in red in Figure 5d. The size of these differences, as well as the lack of improvement in the goodness-of-fit using the multisphere superposition code, indicate that Mie superposition is an appropriate approximation. We conclude that the smaller $R^{2}$ values for this system stem from physical effects-such as weak 

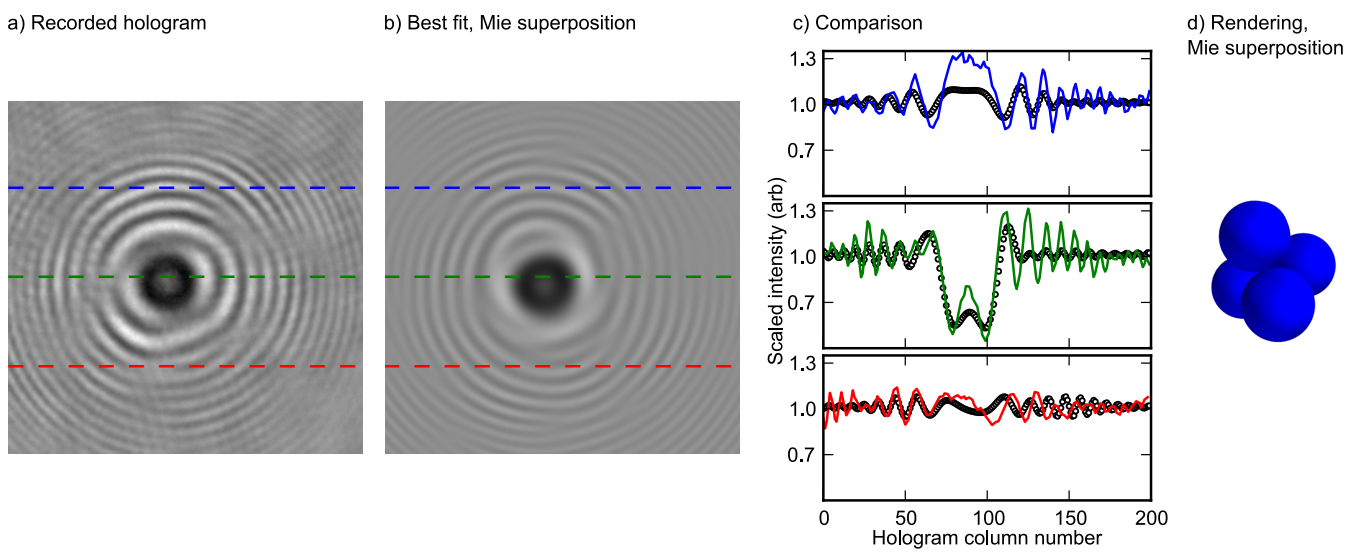

Figure 6: Fit of polytetrahedron hologram in Figure 4c performed using Mie superposition. a) Recorded hologram (shown again for ease of comparison). b) Best-fit model determined from Mie superposition. c) Comparison between recorded hologram (solid lines) and Mie superposition model (open symbols) along the dashed lines in the holograms. d) Rendering showing cluster orientation determined by Mie superposition fit. The incident light propagates into the page.

scattering by the decane droplet - that neither multisphere superposition nor Mie superposition account for.

Further insight into the applicability of Mie superposition comes from examining the particle showing the largest coordinate difference along the optical axis. As shown in Figure 5d, the largest difference occurs when two particles nearly occlude one another. In such a configuration, the assumption that the field incident on each sphere is simply the illuminating plane wave is clearly invalid, as the colloidal spheres scatter most strongly in the forward direction. The field incident on the occluded sphere should therefore include a significant component of the scattered field from the first sphere. Whereas the multisphere superposition solution accounts for this multiple scattering effect, Mie superposition does not.

As expected, Mie superposition fails to fit holograms from the clusters discussed in Section 4.1, where the constituent spheres have a relative index $m \approx 1.2$ and size parameter $x \approx 8.3$. A fit to the polytetrahedron hologram of Figure 4c using a Mie superposition model yields $\chi_{p}^{2}=5.39 \times 10^{-3}$ and $R^{2}=$ 0.463 (Figure 6). These values are much poorer than the values associated with the multisphere superposition model. Moreover, qualitative differences between the best-fit Mie superposition model and the experimental hologram 
are readily apparent.

To quantify the validity of Mie superposition for calculating hologramsor any other scattering-related physical quantity - of multiple spheres, we propose a dimensionless figure of merit. Mie superposition assumes that the exciting field at any sphere, $\mathbf{E}_{e x}$, is approximately equal to the incident plane wave $\mathbf{E}_{i n c}$. Following the multisphere superposition approach, consider $\mathbf{E}_{e x}$ for any given sphere $i$ to be equal to the sum of the incident plane wave and the scattered waves from every other sphere at $i$ :

$$
\mathbf{E}_{e x, i}=\mathbf{E}_{i n c}+\sum_{j \neq i}^{N} \mathbf{E}_{s c a t, j} .
$$

For Mie superposition to be valid, $\left|\mathbf{E}_{s c a t, j}\right|$ must be much smaller than $\left|\mathbf{E}_{\text {inc }}\right|$. This requires the particles to be far enough apart that their near fields do not couple. Then, $\left|\mathbf{E}_{\text {scat }, j}\right|$ scales approximately as $\left|\mathbf{E}_{\text {inc }}\right| S / k R$, where $R$ is a typical interparticle distance and $S$ denotes the magnitude of the amplitude scattering matrix of sphere $j$ in the Mie solution. From the optical theorem, in the Mie solution the extinction efficiency $Q_{\text {ext }}$ for a single sphere is given by

$$
Q_{\text {ext }}=\frac{4}{x^{2}} \Re[S(0)]
$$

where $S(0)$ is the complex amplitude scattering matrix evaluated in the forward direction [25]. Then, if $Q_{e x t} x^{2} / k R \ll 1,\left|\mathbf{E}_{s c a t, j}\right| \ll\left|\mathbf{E}_{i n c}\right|$, and Mie superposition should be valid.

Taking the fitted drop diameter of $4.35 \mu \mathrm{m}$ as a typical interparticle spacing for PMMA spheres like those in the droplet experiments, we find $Q_{\text {ext }} x^{2} / k R=0.11$, in agreement with our previous conclusion that the Mie superposition approximation is accurate for this sample. However, if we consider the sphere shown in red in Figure $5 \mathrm{~d}$, using the nearest-neighbor distance of $1.19 \mu \mathrm{m}$ for $R$ yields $Q_{e x t} x^{2} / k R=0.35$. This larger value indicates that Mie superposition is a poorer approximation for this particle, as borne out by the $150 \mathrm{~nm}$ difference in the fitted position from Mie superposition and multisphere superposition. In contrast, for the polystyrene spheres used in the cluster experiments, which are separated from each other by approximately a particle diameter, $Q_{e x t} x^{2} / k R=13$. We therefore conclude that $Q_{\text {ext }} x^{2} / k R$ should be approximately 0.1 or smaller for Mie superposition results to be trusted to a precision of $100 \mathrm{~nm}$ or better. 
When $Q_{e x t} x^{2} / k R \ll 1$ and $k R$ is large, Mie superposition is not only valid but advantageous compared to multisphere superposition. On a desktop equipped with a $3 \mathrm{GHz}$ Intel Core Duo processor, computing a $256 \times 256$ hologram for the configuration of particles illustrated in Figure 5d requires 11.8 seconds for multisphere superposition and 3.0 seconds for Mie superposition. In contrast, computing the $200 \times 200$ hologram of the six-particle polytetrahedron of Figure 4c requires 3.9 seconds for multisphere superposition and 1.8 seconds for Mie superposition. Here multisphere superposition is only 2 times slower, an acceptable penalty given that the Mie superposition fit fails.

\subsection{Improving the Fits}

Our values of $\chi_{p}^{2}$ are larger than the expected noise level. This indicates that our modeling and fitting procedures, rather than instrumental noise, limit the goodness of our fits. Challenges for future work fall into two categories: modeling additional physical phenomena, and properly optimizing model parameters to determine a best fit.

Our current hologram models do not capture several physical effects. First, while we choose immersion fluids for our microscope objective lenses to index-match the continuous phases of our samples as closely as possible, we cannot completely eliminate spherical aberration. Second, although we have fit for only a single, average particle radius in these experiments, no colloidal suspension is perfectly monodisperse. Third, while the well depth of the depletion attraction in the clusters is at least several $k_{B} T$, thermally-induced vibrations can perturb the cluster structures. We are currently working to model and account for spherical aberration in our hologram analysis. Fitting for individual particle positions in a cluster, rather than assuming geometric regularity, may address the latter two phenomena.

Local minima in the fitting landscape pose another challenge to obtaining good fits. The number of local minima may increase with the number of parameters in the hologram models. This is partly why we constrain all the spheres in our scattering models to be the same size. However, incorporating additional physical parameters into our hologram models will be necessary to study more complex scatterers, such as nonrigid clusters of weakly interacting spheres. Consequently, in order to prevent fits from becoming trapped in local minima, we are currently exploring the use of other minimizers, including one based on the $r$-algorithm [26]. 


\section{Conclusions}

We have demonstrated that superposition solutions are accurate models for fitting digital holograms of up to six colloidal spheres. The multisphere superposition solution can reproduce the holograms of clusters in which there is significant coupling of the near-fields between spheres. We have also introduced a faster computational tool, Mie superposition, that should facilitate the study of multisphere systems where the particles are far apart. This approximate technique is accurate when $Q_{\text {ext }} x^{2} / k R \ll 1$.

The six-particle clusters we have imaged are large enough to show nontrivial dynamics related to multiple ground states [9]. We have also imaged configurations of spheres without a fixed geometry. Our experiments are thus the first step towards measuring internal rearrangements and self-assembly of sphere clusters. Moreover, analysis of dynamical information obtained from time series of holograms like those studied in Figure 5 may yield insight into colloidal interactions at liquid-liquid interfaces.

\section{Acknowledgments}

We thank Andrew B. Schofield for synthesizing PMMA microspheres, and Keith A. Brown for assistance with the fabrication of NDEP devices. Rebecca W. Perry and Thomas G. Dimiduk acknowledge the support of National Science Foundation (NSF) Graduate Research Fellowships. This work was funded by the NSF through CAREER grant no. CBET-0747625 and through the Harvard MRSEC, grant no. DMR-0820484. Computations were performed on the Odyssey cluster, managed by the Harvard FAS Sciences Division Research Computing Group. NDEP device fabrication was performed at the Center for Nanoscale Systems (CNS), a member of the National Nanotechnology Infrastructure Network (NNIN), which is supported by the NSF under grant no. ECS-0335765. CNS is part of Harvard University.

\section{References}

[1] S. H. Lee, Y. Roichman, G. R. Yi, S. H. Kim, S. M. Yang, A. van Blaaderen, P. van Oostrum, D. G. Grier, Characterizing and tracking single colloidal particles with video holographic microscopy, Opt. Express 71 (2005) 041106. 
[2] F. C. Cheong, S. Duarte, S. Lee, D. G. Grier, Holographic microrheology of polysaccharides from streptococcus mutans biofilms, Rheol. Acta 48 (1) (2008) 109-115.

[3] F. Cheong, K. Xiao, D. Grier, Technical note: Characterizing individual milk fat globules with holographic video microscopy, J. Dairy Sci. 92 (1) (2009) 95-99.

[4] K. Xiao, D. G. Grier, Multidimensional optical fractionation of colloidal particles with holographic verification, Phys. Rev. Lett. 104 (2) (2010) 028302 .

[5] K. Xiao, D. G. Grier, Sorting colloidal particles into multiple channels with optical forces: Prismatic optical fractionation, Phys. Rev. E 82 (5) (2010) 051407.

[6] J. Fung, K. E. Martin, R. W. Perry, D. M. Kaz, R. McGorty, V. N. Manoharan, Measuring translational, rotational, and vibrational dynamics in colloids with digital holographic microscopy, Opt. Express 19 (9) (2011) 8051-8065.

[7] D. M. Kaz, R. McGorty, M. Mani, M. P. Brenner, V. N. Manoharan, Physical ageing of the contact line on colloidal particles at liquid interfaces, Nat. Mater. 11 (2012) 138-142.

[8] D. W. Mackowski, M. I. Mishchenko, Calculation of the T matrix and the scattering matrix for ensembles of spheres, J. Opt. Soc. Am. A 13 (11) (1996) 2266-2278.

[9] G. Meng, N. Arkus, M. P. Brenner, V. N. Manoharan, The free-energy landscape of clusters of attractive hard spheres, Science 327 (5965) (2010) 560-563.

[10] R. McGorty, J. Fung, D. Kaz, S. Ahn, V. N. Manoharan, Measuring dynamics and interactions of colloidal particles with digital holographic microscopy, in: Digital holography and three-dimensional imaging, OSA Technical Digest (CD), Optical Society of America, 2008, p. DTuB1.

[11] M. G. Nikolaides, A. R. Bausch, M. F. Hsu, A. D. Dinsmore, M. P. Brenner, C. Gay, D. A. Weitz, Electric-field-induced capillary attraction 
between like-charged particles at liquid interfaces, Nature 420 (6913) (2002) 299-301.

[12] K. J. Mysels, P. Mukerjee, Critical micelle concentrations of aqueous surfactant systems, NSRDS-NBS, 36, U.S. National Bureau of Standards, Washington, 1971.

[13] S. Asakura, F. Oosawa, Interaction between particles suspended in solutions of macromolecules, J. Polymer Sci. 33 (126) (1958) 183-192.

[14] A. Vrij, Polymers at interfaces and the interactions in colloidal dispersions, Pure Appl. Chem. 48 (4) (1976) 471.

[15] E. M. Herzig, K. A. White, A. B. Schofield, W. C. K. Poon, P. S. Clegg, Bicontinuous emulsions stabilized solely by colloidal particles, Nat. Mater. 6 (12) (2007) 966-971.

[16] L. Antl, J. Goodwin, R. Hill, R. Ottewill, S. Owens, S. Papworth, J. Waters, The preparation of poly(methyl methacrylate) latices in nonaqueous media, Colloids. Surf. 17 (1) (1986) 67-78.

[17] G. H. Markx, R. Pethig, J. Rousselet, The dielectrophoretic levitation of latex beads, with reference to field-flow fractionation, J. Phys D: Appl. Phys. 30 (17) (1997) 2470-2477.

[18] Y. Huang, X. Wang, F. Becker, P. Gascoyne, Introducing dielectrophoresis as a new force field for field-flow fractionation, Biophys. J. 73 (2) (1997) 1118-1129.

[19] H. Morgan, A. G. Izquierdo, D. Bakewell, N. G. Green, A. Ramos, The dielectrophoretic and travelling wave forces generated by interdigitated electrode arrays: analytical solution using fourier series, J. Phys. D: Appl. Phys. 34 (10) (2001) 1553-1561.

[20] P. L. Biancaniello, J. C. Crocker, Line optical tweezers instrument for measuring nanoscale interactions and kinetics, Rev. Sci. Inst. 77 (2006) 113702.

[21] T. M. Kreis, Frequency analysis of digital holography with reconstruction by convolution, Opt. Eng. 41 (8) (2002) 1829-1839. 
[22] M. I. Mishchenko, L. D. Travis, D. W. Mackowski, T-matrix computations of light scattering by non-spherical particles: a review, J. Quant. Spectrosc. Radiat. Transfer 55 (5) (1996) 535576.

[23] K. A. Fuller, D. W. Mackowski, Electromagnetic scattering by compounded spherical particles, in: M. I. Mishchenko, J. W. Hovenier, L. D. Travis (Eds.), Light scattering by nonspherical particles: Theory, measurements, and applications, Academic Press, 2000, pp. 225-272.

[24] W. Mendenhall, T. Sincich, Statistics for engineering and the sciences, 3rd Edition, Dellen Pub. Co., San Francisco, 1991.

[25] C. F. Bohren, D. R. Huffman, Absorption and scattering of light by small particles, Wiley-Interscience, 1998.

[26] N. Z. Shor, N. G. Zhurbenko, A minimization method using the operation of extension of the space in the direction of the difference of two successive gradients, Cybernetics 7 (3) (1974) 450-459. 